\title{
Effects of table tennis multi-ball training on dynamic posture control
}

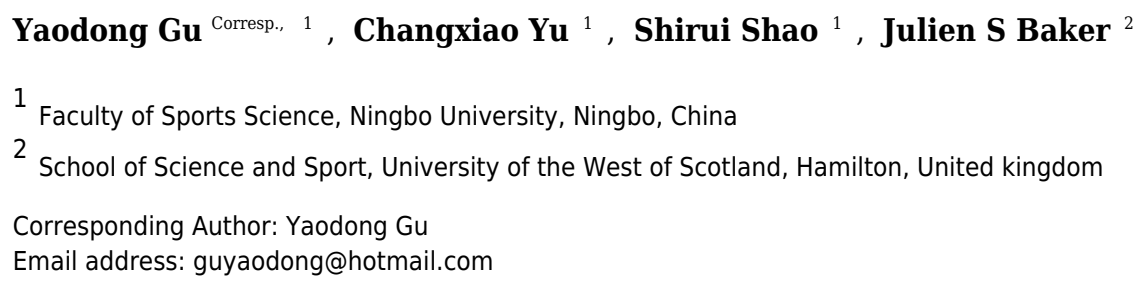

Background. Prior to the 2017 table tennis season, each participant performed the anterior, posteromedial, and posterolateral the star excursion balance test (SEBT) reach distances in a randomized order. The aim of this study was to assess the effects of table tennis multi-ball training and dynamic balance on performance measures of the SEBT for the male and female. Methods. The limb lengths of the 12 table tennis athletes were measured bilaterally in the study. Besides warm-up end, the data of this study were recorded at a regular interval at approximately 16 minutes for the entire multi-ball training session, and they were defined as Phase I, Phase II, Phase III, respectively. The Borg rating of perceived exertion (RPE) scale was used to document the degree of physical strain.

Results. Reaching distances showed a decrease with training progression in all directions. Compared with the male table tennis athletes, the females showed poorer dynamic posture control, particularly when the free limb was considered with the right-leg stance toward posterolateral and posteromedial directions in phase I. Discussion. This study suggests that during table tennis multi-ball training the male should have a regulatory protocol to compensate the deficit observed in phase II, but the females should be given the protocol in phase I. 
1

2

3

4

5

6

7

8

9

10

11

12

13

14

15

16

17

18

19

20

21

22

\section{Effects of table tennis multi-ball training on dynamic posture control}

b School of Science and Sport, University of the West of Scotland, Hamilton, UK

\section{*Corresponding Author: Yaodong Gu}

E-mail Address: guyaodong@hotmail.com

1 Address: Faculty of Sports Science, Ningbo University, No. 818, Fenghua Road, Jiangbei

District, Ningbo, Zhejiang Province, China.

3




\section{Effects of table tennis multi-ball training on dynamic posture control}

\section{Abstract}

Background. Prior to the 2017 table tennis season, each participant performed the anterior, posteromedial, and posterolateral the star excursion balance test (SEBT) reach distances in a randomized order. The aim of this study was to assess the effects of table tennis multi-ball training and dynamic balance on performance measures of the SEBT for the male and female.

Methods. The limb lengths of the 12 table tennis athletes were measured bilaterally in the study. Besides warm-up end, the data of this study were recorded at a regular interval at approximately 16 minutes for the entire multi-ball training session, and they were defined as Phase I, Phase II, Phase III, respectively. The Borg rating of perceived exertion (RPE) scale was used to document the degree of physical strain.

Results. Reaching distances showed a decrease with training progression in all directions. Compared with the male table tennis athletes, the females showed poorer dynamic posture control, particularly when the free limb was considered with the right-leg stance toward posterolateral and posteromedial directions in phase I .

Discussion. This study suggests that during table tennis multi-ball training the male should have a regulatory protocol to compensate the deficit observed in phase $\mathbb{I}$, but the females should be given the protocol in phase I . 


\section{Introduction}

Table tennis is one of the most popular sports in the world. According to a report by the International Sports Federation, the population of table tennis participants has reached over 300 million worldwide (Zhang, 2017). Playing table tennis is regarded as a pro-health sporting pastime, which is generally accepted by more and more people who engage in physical activity (Biernat, Buchholtz \& Krzepota, 2018). As a rational movement, the characteristics of table tennis play, which involve complex spatial movements of the body that include, acceleration, deceleration, direction change, moving quickly and balance all help players generate optimum stroke production (Girard \& Millet, 2009). Table tennis coaches have observed the importance of conditioning and have been proactive in helping table tennis players achieve a better competitive condition. To be successful in the sport, table tennis players are usually asked to perform high intensity training in the preseason as part of their physiological preparation. In addition, technical practice is essential for table tennis players in training sessions, especially during the preseason phase. In order to develop the so-called dynamic stereotype, the table tennis player should try to improve the skills involved when stroking the ball that is in a fixed position or with tactical variations. As a common training method for technical practice, multi-ball training requires the players to repeatedly stroke and return the balls with a combination of feasible footwork (Zhang, 2017), which is generally applied in training sections.

To our knowledge, dynamic balance and fatigue are related (Gioftsidou, Malliou, Pafis, Beneka \& Godolias, 2011), and the greater body control for individuals would possess a lower risk of injuries during dynamic movements (Kollock, 2018; Knapik, Cosiolima, Reynolds \& Shumway, 2015). The star excursion balance test (SEBT) is a reliable, valid and easy method to measure 
lower-limb function in sports and clinical practice (Munro \& Herrington, 2010; Hertel, Miller \& Denegar, 2000; Kinzey \& Armstrong, 1998; Gribble \& Hertel, 2003a; Bouillon \& Baker, 2011). The intra class correlation coefficient values of the SEBT have ranged from 0.67 to 0.96 , with high intra rater reliability values of 0.81 to 0.93 (Hertel et al., 2000; Kinzey \& Armstrong, 1998). Therefore, the SEBT has wide practical application in assessing the ability to maintain balance in a single-leg stance in exercise testing, rehabilitation and training (Winter, Patla \& Frank, 1990; Robinson \& Gribble, 2008). For example, the test has not only been used previously to predict lower limb injury in high school soccer (Bressel, Yonker, Kras \& Heath, 2007; Filipa, Byrnes, Paterno, Myer \& Hewett, 2010; Rasool \& George, 2007) and basketball (Bressel et al., 2007; Plisky, Rauh, Kaminski \& Underwood, 2006), but also used for convalescent patients with chronic ankle instability (Olmsted, Carcia, Hertel \& Shultz, 2002; Gribble, Hertel, Denegar \& Buckley, 2004; Hertel, Braham, Hale \& Olmsted-Kramer, 2006; Hubbard, Kramer, Denegar, \& Hertel, 2007; Isles, Choy, Steer \& Nitz, 2004) and anterior cruciate ligament injuries (Herrington, Hatcher, Hatcher \& McNicholas, 2009). In addition, it has been applied to evaluate the effects of patellar taping on lower-limb kinematics and dynamic postural control (Aminaka \& Gribble, 2008). As a closed-kinetic chain exercise, the SEBT mimics the single-leg squat exercise while attempting maximal reach with the opposite leg, which requires better neuromuscular control, flexibility, balance and strength for the stance leg (Olmsted et al., 2002). Indeed, the SEBT was not only used as a method of assessment, but also as an approach used to improve the performance of movement skills in an athletic population (Munro \& Herrington, 2010; Filipa et al., 2010). In addition, a previous study showed that males have larger reach distance when compared with females (Gribble, Hertel \& Piegaro, 2003b). However, there was no significant gender differences observed when the reach distances were normalized (Gribble, Robinson, Hertel \& Denegar, 2009). In addition, Day and colleagues (2004) corroborated that the rating of perceived exertion (RPE) is a reliable method to quantify exercise intensities during highintensity, moderate-intensity and low-intensity resistance training. Faulkner et al. (2008) found a relationship between the RPE scales and the exercise time during competitive running races. 
97 Borg (1982) showed that the scale values, range from 6 to 20 can be used to denote heart rates

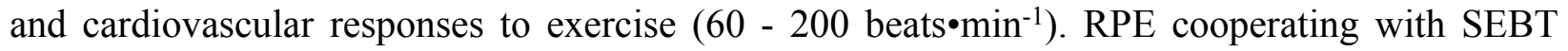
measures the effect of table tennis multi-ball training on dynamic posture control from subjectivity to objectivity for this study.

Dynamic postural-control tasks require contributions from the kinetic chain to generate a greater coordinated movement pattern (Winter et al., 1990; Gribble et al., 2009). As a source of energy, the lower limb can transfer energy upwards to the upper limb through the kinetic chain (Elliott, 2006; Qian, Zhang, Baker \& Gu, 2016). Previous studies reported that lower limb drive was the origin of the kinetic chain, which not only impacts on the quality of skills and tactics used in table tennis (Qian et al., 2016; Fu et al., 2016), but also in lawn tennis (Elliott, 2006; Girard, Micallef \& Millet, 2005). These studies showed that power lower-limb drive has an important relationship with optimizing energy transfer in the kinetic chain, which influence the lower-limb flexibility and tactical application. In order to hit back balls from different directions, table tennis players need to be able to adjust their body into a apposite position that facilitates ball return. Moreover, as a multiple-set sport, table tennis players should be able to distribute their energy demand in a way that facilitates continuous strokes and good movement patterns during competition and training. Due to the onset of fatigue, there is a negative relationship between sport performance and playing time (Yaggie \& Armstrong, 2004; Pau, Ibba \& Attene, 2014). It's well known, the movement of the center of pressure (COP) is a practical parameter used to assess lower extremity performance. Yu et al. (2018) analyzed the trends of load transmission on the foot between squat and standing serves in female table tennis athletes, according to the investigations of COP trajectory. They found squat serve needs higher lower-limb drive compared with standing serve during a short serve. In fact, potential mechanisms that limit performance have been reported, as being related to increasing muscle-spindle discharge that influence the feedback to the central nervous system and then decrease postural control (Gribble et al., 2009; Rozzi, Yuktanandana, Pincivero \& Lephart, 2000; Khin-Myo-Hla, Sakane \& Hayashi, 1999). However, to date no study has looked at multi-ball training associated with 
124 SEBT and examined what percentage change reflects the degree of exercise intensity that would 125 impact on the performance of table tennis preparation preseason. In addition, there is no study 126 that report the effects of neuromuscular fatigue on the performance of the dynamic postural127 control task during table tennis multi-ball training. The aims of the current study are firstly, to 128 identify any relationship between multi-ball training and dynamic postural control in table tennis 129 players; secondly, to provide a guide to formulate feasible pacing strategy for table tennis 130 coaches and players. The research hypotheses of the study were that (1) the normalized reach 131 distance would show decrease when both of the groups perform the SEBT with continuous 132 multi-ball training, and the significant decrease in reach distance would occur later in the male 133 table tennis players compared with the female players, (2) compared with warm-up end, the 134 reach distance for both the dominant and non-dominant leg in the male and female would show 135 different conditions with the training continuing and (3) as training continues, the COP motion 136 (medial-lateral direction, length-X; anterior-posterior direction, length-Y) would show 137 significant changes compared with warm-up end.

\section{Methods}

\section{Participants}

141

142

143

Twelve experts $(6$ males: age $21.6 \pm 1.42$ years, weight $73.75 \pm 3.24 \mathrm{~kg}$, height $1.78 \pm 0.04 \mathrm{~m}$, training experience $14.2 \pm 1.46$ years; 6 females: age $21.6 \pm 1.53$ years, weight $63.72 \pm 5.4 \mathrm{~kg}$, height $1.65 \pm 0.05 \mathrm{~m}$, training experience $14.2 \pm 1.62$ years) from Ningbo University table tennis team volunteered to participate in the study. All participants were National Division I players, they were right-handed and free from any previous lower limb injuries, surgery, foot diseases and had no previous injury for at least six months. The Ethics Committee of Ningbo University (RAGH20170819) has approved this study, and written informed consent was obtained from all individuals prior to participation. No participant received any payment for this study. 
149 In this study, dominant lower limb was determined according to the ball-kick test (Zakas, 2006).

150 The participants were asked to kick football with arbitrary power and maximal accuracy through a

151 set of obstacles placed $1 \mathrm{~m}$ apart and $10 \mathrm{~m}$ from the participants, the limb used to kick the football

152 was regarded as the dominant limb and the other side was non-dominant limb.

\section{Procedures}

154 This test was executed at Ningbo University table tennis training gymnasium. The study was divided into three sessions, as shown in Figure 1, each session comprised four phases (warm-up

156

157

158 end, Phase I, Phase II, Phase III). The time interval of two sessions for this trial was over 24 hours. The first two sessions consisted of a familiarization session that included an instructional video, informed-consent the test procedures, introduction on the Borg 6-20 RPE scale and explanation of how to use the scale. At the third session, each participant was asked to rate his/her perceived exertion based on the RPE scale by answering the question "How was your workout?", the scale is shown in Figure 2.

Before the trials, each participant was given a standardized warm-up of $20 \mathrm{~min}$ in the experimental environment. Participants were then assigned multi-ball training protocols in pairs, which included change of direction at impact cooperating topspin, backspin etc. All data sets from the study were recorded at each phase (each phase lasted approximately 16 minutes) for one entire training session (Malliou et al., 2008). Participants performed the SEBT in the four different training conditions (warm-up end, Phase I, Phase II, Phase III), respectively. Participants were asked to single-leg stand in the center of a grid, then using the free limb to reach in the anterior, posteromedial and posterolateral directions touching lightly - so as not to aid balance - and then return the starting position. The time of one entire session was approximately 50 minutes. The reach distance (with the big toe as the dependent measure for participants) was recorded when the players could maintain not less than 30 seconds in each direction. The test was performed from the left leg support to the right leg, and both lower limbs were measured under the same conditions respectively. Each participant performed eight times in each direction. The mean of the top five 
175

176 (Figure 3).

177 At the initial session, to calculate the dependent variable for normalized reach distance, leg length

reach distances for each participant in the three reach directions were used for further data analysis was measured with the participants in supine position. The participants' right and left leg lengths were measured (respectively), from the anterior superior iliac to the distal end of the medical malleolus with a calibrated tape measure.

The trial was discarded and repeated when: (1) the stance leg lost balance, (2) the heel of the stance leg lost contact, (3) the support time was less than required, and (4) the reaching foot could not return the starting position.

\section{Insert Figure 1}

Insert Figure 2

Insert Figure 3

\section{Instrumentation}

COP was recorded by a Novel Pedar insole plantar pressure measurement system (Novel GmbH, Munich, Germany) at a frequency of $50 \mathrm{~Hz}$. This equipment has been previously used in kinetic analysis for table tennis and tennis (Girard, Eicher, Micallef \& Millet, 2010; Fu et al., 2016; Qian et al., 2016). The length of COP motion includes length-X and length-Y, which are routinely used to assess postural control (Fu et al., 2016). Measuring insoles were placed bilaterally inside the participants' shoes (size $38-42 \mathrm{~cm}$ ), and the data recording was sampled through Bluetooth technical equipment. This equipment did not influence technical motions, and was worn in both the training times and the trial processes, respectively.

\section{Statistical Analysis}

All statistical tests were performed using SPSS version 19.0 software (SPSS Inc., Chicago, IL, USA) for Windows. Prior to statistical comparisons, an initial Shapiro-Wilks test confirmed that 
199 all data were normally distributed. Descriptive statistics were used to calculate the means \pm 200 standard deviations for all participants. To examine the differences in the reach distance of 201 dominant and non-dominant limbs during the SEBT for the male or the female, independent $t$ 202 tests were carried out, respectively. There were pairwise comparisons in two different phases for 203 the RPE, reach distance and length of COP (-X and -Y), one-way repeated-measures analysis of 204 variance (ANOVA) was used to determine differences in the four training stages. The 205 significance level for all tests was set at $p \leq 0.05$. The effect size was determined based on 206 Cohen's d which was used to compare the differences in the average of the two groups. Effect 207 size (ES) is evaluated as trivial $(\geq 0.19)$, small $(\geq 0.2$ and $\leq 0.49)$, medium $(\geq 0.50$ and $\leq 0.79)$ 208 and large $(\geq 0.80)$, respectively (Cohen, 1992).

209

210

\section{Results}

\section{RPE}

212 Descriptive characteristics of the participants in the four phases are presented in Table 1. As 213 expected, significant differences were found in phase I, phase II and phase III compared with 214 warm-up (Figure 4).

Insert Figure 4

Insert Table 1

\section{SEBT}

As Figure 5 shown, there were similar high between left and right legs in reaching distances when performed the SEBT for the two groups. Based on independent t-tests, the reaching distance had no

221 significant differences in the three reach directions of the each phase between dominant and non222 dominant limbs for the male or female $(p>0.05)$ (Figure 5). In addition, compared with warm-up, 
223 the reach distance of the right leg for the male participants at phase $\mathbb{I}$ showed significant decrease

224 in the anterior direction and posteromedial direction (Table 2,3). The reach distance of the left leg

225 for the male participants at the warm-up end showed significantly greater distance in the

226 posterolateral direction and posteromedial direction than phase II and III, in addition, greater

227 distances were recorded in the anterior direction compared with the phase III (Table 2,3). For the

228 female participants, there were no significant changes in the all reach distances observed for the

229 right leg except the posterolateral direction at phase $\mathbb{I}$ and $\mathbb{I I}$ when compared with warm-up end 230 (Table 2,4). However, for the reach distance of the left leg, there were significant differences in all

231 directions except the anterior direction at phase I when compared with warm-up (Table 2,4).

232 Insert Figure 5

233 Insert Table 2

$234 \quad$ Insert Table 3

235 Insert Table 4

236

237 COP

238 Figure 6 displays the length of COP in each phase. For all participants, length-X increased more 239 than warm-up end, but length-Y decreased more compared with warm-up end following 240 continuous training when performed the SEBT in all directions $(p \leq 0.05)$. For the male 241 participants, when the right lower limb reached anterior and posteromedial directions, length-X 242 of the support leg in phase II was significantly greater, but length-Y was significantly smaller 243 than warm-up end $(p \leq 0.05)$. Similarly, compared with warm-up end, length-X of the right leg 244 was significantly greater in phase II and III when the free limb reached 245 posteromedial/posterolateral and anterior directions (respectively), but length-Y was 
246 significantly smaller. For female participants, the length-X of the left leg was significantly

247 greater in phase $\mathbb{I}$ than warm-up end when the free limb reached posterolateral direction, but

248 length-Y was significantly smaller in the same phase. In addition, compared with warm-up end,

249 length-X of the right leg was significantly greater. The free leg reached the anterior direction in

250 phase II and it reached the posteromedial/posterolateral direction in phase I, but length-Y were

251 significantly smaller.

252 Insert Figure 6

\section{Discussion}

255 To our knowledge, this is the first study that has provided evidence related to the relationship 256 between dynamic balance and multi-ball training in preseason table tennis. The purpose of this 257 study was to assess the effects of multi-ball training on dynamic posture control using the SEBT during table tennis multi-ball training. Based on the findings of the study, the results and analysis could help coaches to better regulate the training pace during preseason preparation. As expected, the results of the study indicated that the significant decrease in dynamic balance mainly occurred in phase $\mathbb{I}$, but the female participants started to decline earlier than the males. Additionally, when postural control started to decrease significantly, length-X of COP motion showed to increase more than that observed at the warm-up end. The length-Y of COP motion showed significant reductions when the same stages were compared.

It is well documented that the Borg RPE scale has become a standard method to evaluate perceived exertion and has been used in exercise science to quantify exercise intensity (Day et al., 2004; Noble, Borg, Jacobs, Ceci \& Kaiser, 1983; Noble \& Robertson, 1996). In addition, Foster et al. $(1996 ; 1998 ; 2001 ; 2017)$ studied the specific stages of exercises in an entire aerobic exercise session using the RPE scale. They provided evidence that this method can be applied to 
270 quantifying the intensity of exercise during various types of exercise. The results of our study 271 found that there were apparently different training loads between each phase for both the male 272 and female participants. This provided useful information for comparative purposes between the 273 male and female participants. Considering the characteristics of table tennis multi-ball training,

274

275

276

277

278

279

280

281

282

283

284

285

286

287

288

289

290

291

292

293

294

295

296 this finding may be related to the training time observed and the duration of the activity may be contributing to the fatigue profiles recorded. However, because we only used one method it may be speculative to try and explain the relationship between dynamic balance and training time. Therefore, further work is needed to examine in detail any relationship observed.

Plisky et al. (2006) have indicated that potential risk factors for injury may result in the opposite limb if a reduced reach distance in the free limb is observed when using the measurement method employed in the SEBT. Previous studies have indicated that the reduced work of skeletal muscle is associated with muscle fatigue, which was believed to be a potential cause of increased injury rates for athletes during unexpected perturbations (Hassanlouei, Arendt-Nielsen, Kersting \& Falla, 2012). Additionally, in racket sport players, there is a potential source of muscle asymmetry between two legs, which could largely affect the pattern of movement and perhaps induce sport injuries (Ye et al., 2018; Lam, Fan, Zheng \& Lee, 2018; Ireland et al., 2013; Sanchis-Moysi et al., 2010). The less adept lower limb for balance, the greater the inability to provide an optimally stable base for the neuromuscular system to maintain a constant tension. This could promote absorption of increased load and contribute to the instability recorded (Fang, 2018; Plisky et al., 2006; Hewett, Myer \& Ford, 2001; Weaver, Mueller, Kalsbeek \& Bowling, 1999). In addition, Earl and Hertel (2001) showed that the support leg muscles could be activated to a different extent during the SEBT, which could be contributing to postural sway under during continuous table tennis multi-ball training. Exercise-induced fatigue would result in failure to produce maximal force and reduce flexibility, which could influence motor performance. Previous studies have corroborated that fatigue-induced impairments could badly impact on joint proprioception and neuromuscular control (Hassanlouei et al., 2012; Voight, Hardin, Blackburn, Tippett \& Canner, 1996; Ribeiro, Santos, Goncalves \& Oliveira, 2008; Miura et al., 2004). 
297 Boden et al. (2000) reported that these findings constantly occurred at the end stages of athletic

298

299

300

301

302

303

304

305

306

307

308

309

310

311

312

313

314

315

316

317

318

319

320

321

322

competition. Additionally, the motions of sudden deceleration, landing and pivoting maneuvers throughout the entire table tennis multi-ball training regime would exacerbate the risk of sport injury. In order to try and quantify the influence of postural control on a participant performance, Caron er al. (2000) measured the displacement of the COP. The findings from our study in relation to COP displacement indicated that the reach distance in nearly all directions was beginning to show a sharp decline trend in phase $\mathbb{I}$. At the same time, in order to maintain posture balance, the length-X of the COP showed increases, but its length-Y showed a decrease when compared with the warm-up end. The results indicated that dynamic balance was significantly decreased with table tennis multi-ball training. When we consider the step features in table tennis, that include suddenly stopping and pivoting, which place repeated rotational shear and loading forces on each joint of the lower limbs during table tennis multi-ball training, it may be useful to compare the actions with tennis players. Tennis players are at risk of increasing overuse and acute injuries including ligament sprains, chronic muscle strain, hamstring strains, stress fractures, ankle sprains, shin splints, knee contusions and growth plate injuries (Murphy, Connolly \& Beynnon, 2003; Kibler \& Safran, 2005; Hutchinson, Laprade, Burnett, Moss \& Terpstra, 1995). According to the report of Kondrič et al. (2011), they found that the most frequent injuries in racket sports is muscle tissues from training and/or competition processes, and there is a high percentage of injuries in lower-limb joints (ankle and foot in particular; $23.69 \%$ in total). They also indicated that due to the characteristics of abrupt blocking movements in playing table tennis, the percentage of hip injuries exists $5.76 \%$. In relation to these findings since table tennis players exhibited a apparent decline in dynamic balance in phase II, a sport injury prevention program may be beneficial.

As a method of neuromuscular training, the SEBT can be incorporated into a pre-participation physical examination for athletes to improve identified specific deficits in the preseason phase of competition (Plisky et al., 2006). Paterno et al. (2004) and Holm et al. (2004), reported that a 
323

324

325

326

327

328

329

330

331

332

333

334

335

336

337

338

339

340

341

342

343

344

345

346

347

period of 6-7 weeks of balance training would help athletes rapidly improve postural stability. Based on the finding of this study, these methods should be incorporated into preseason training and preparation phases. Future work would include training monitoring being dominated by emerging new technologies that would consist of real-time monitoring of the internal and external forces during training and recovery (Foster et al., 2017). Therefore, the RPE and the SEBT may be merged to formulate exciting training monitoring design via new and exciting technologies.

Some limitations to this study should be noted. Firstly, the data were based on one table tennis season using one university table tennis team. Although all participants were granted with National Division I status, it may limit the external validity at some degree. Further, the study did not collate lower-limb strength and flexibility data. There is another potential limitation is that we did not have electromyographic data to analyze the internal mechanism of inducing decrease in dynamic balance from the standpoint of neuromuscular system. In addition, future studies should consider research during competition to assess the relationship between dynamic balance, strength and time duration.

\section{Conclusions}

This study examined the relationship between dynamic posture control and the time of table tennis multi-ball training preseason. The study's prospective design allowed the requirements of each trainee to reduce the risk of injury during table tennis multi-ball training. Further, the findings of this study may assist the coaches and the certified athletic trainees to understand the effects of table tennis multi-ball training on dynamic posture control. The findings may also be useful in determining musculoskeletal deficits in performance. Finally, the findings may also help in the design and implementation of specific rehabilitative programs when the postural control descends significantly. 


\section{References}

Aminaka N, Gribble PA. 2008. Patellar taping, patellofemoral pain syndrome, lower extremity kinematics, and dynamic postural control. Journal of athletic training 43(1): 21-28.

Biernat E, Buchholtz S, Krzepota J. 2018. Eye on the Ball: Table Tennis as a Pro-Health Form of Leisure-Time Physical Activity. International journal of environmental research and public health 15(4): 738.

Boden BP, Dean GS, Feagin JA, Garrett WE. 2000. Mechanisms of anterior cruciate ligament injury. Orthopedics 23(6): 573-578.

Borg G. 1970. Perceived exertion as an indicator of somatic stress. Scandinavian journal of rehabilitation medicine 2(2): 92-98.

Borg GA. 1982. Psychophysical bases of perceived exertion. Medicine and science in sports and exercise 14(5): 377-381.

Bouillon LE, Baker JL. 2011. Dynamic balance differences as measured by the star excursion balance test between adult-aged and middle-aged women. Sports health 3(5): 466-469.

Bressel E, Yonker JC, Kras J, Heath EM. 2007. Comparison of static and dynamic balance in female collegiate soccer, basketball, and gymnastics athletes. Journal of athletic training 42(1): 42-46.

Caron O, Gélat T, Rougier P, Blanchi JP. 2000. A comparative analysis of the center of gravity and center of pressure trajectory path lengths in standing posture: an estimation of active stiffness. Journal of Applied Biomechanics 16(3): 234-247.

Cohen J. 1992. Statistical power analysis. Current directions in psychological science 1(3): 98101.

Day ML, Mcguigan MR, Brice G, Foster C. 2004. Monitoring exercise intensity during resistance training using the session RPE scale. The Journal of Strength \& Conditioning Research 18(2): 353-358.

Earl JE, Hertel J. 2001. Lower-extremity muscle activation during the Star Excursion Balance Tests. Journal of Sport Rehabilitation 10(2): 93-104.

Elliott B. 2006. Biomechanics and tennis. British journal of sports medicine 40(5): 392-396.

Fang Q. 2018. Comparisons of Foot Pressure between Teenager Girls and Young Female Adults. Physical Activity and Health, 2(1), 24-28.

Faulkner J, Parfitt G, Eston R. 2008. The rating of perceived exertion during competitive running scales with time. Psychophysiology 45(6): 977-985.

Filipa A, Byrnes R, Paterno MV, Myer GD, Hewett TE. 2010. Neuromuscular training improves performance on the star excursion balance test in young female athletes. Journal of orthopaedic \& sports physical therapy 40(9): 551-558.

Foster C. 1998. Monitoring training in athletes with reference to overtraining syndrome. Medicine and science in sports and exercise 30: 1164-1168.

Foster C, Daines E, Hector L, Snyder AC, Welsh R. 1996. Athletic performance in relation to training load. Wisconsin medical journal 95(6): 370-374. 
387 Foster C, Florhaug JA, Franklin J, Gottschall L, Hrovatin LA, Parker S, ... Dodge, C. 2001. 388 A new approach to monitoring exercise training. The Journal of Strength \& Conditioning 389 Research 15(1): 109-115.

390 Foster C, Rodriguez-Marroyo JA, de Koning JJ. 2017. Monitoring Training Loads: The Past, 391 the Present, and the Future. International Journal of Sports Physiology and Performance 392 12(Suppl 2): S2-2.

393 Fu F, Zhang Y, Shao S, Ren J, Lake M, Gu Y. 2016. Comparison of center of pressure 394 trajectory characteristics in table tennis during topspin forehand loop between superior and 395 intermediate players. International journal of Sports Science \& Coaching 11(4): 559-565.

396 Gioftsidou A, Malliou P, Pafis G, Beneka A, Godolias G. 2011. Effects of a soccer training 397 session fatigue on balance ability. Journal of Human Sport and Exercise 6(3).

398 Girard O, Millet GP. 2009. Neuromuscular fatigue in racquet sports. Physical medicine and 399 rehabilitation clinics of North America 20(1): 161-173.

400 Girard O, Eicher F, Micallef JP, Millet G. 2010. Plantar pressures in the tennis serve. Journal 401 of sports sciences 28(8): 873-880.

402 Girard O, Micallef JP, Millet GP. 2005. Lower-limb activity during the power serve in tennis: 403 effects of performance level. Medicine \& Science in Sports \& Exercise 37(6): 1021-1029.

404 Gribble PA, Hertel J. 2003a. Considerations for normalizing measures of the Star Excursion 405 Balance Test. Measurement in physical education and exercise science 7(2): 89-100.

406 Gribble PA, Hertel J, Piegaro AB. 2003b. Predictors for performance of dynamic postural 407 control using the Star Excursion Balance Test. Measurement in Physical Education and 408 Exerciser Science 7: 89-100.

409 Gribble PA, Hertel J, Denegar CR, Buckley WE. 2004. The effects of fatigue and chronic 410 ankle instability on dynamic postural control. Journal of athletic training 39(4): 321.

411 Gribble PA, Robinson RH, Hertel J, Denegar CR. 2009. The effects of gender and fatigue on 412 dynamic postural control. Journal of sport rehabilitation 18(2): 240-257.

413 Hale SA, Hertel J, Olmsted-Kramer LC. 2007. The effect of a 4-week comprehensive 414 rehabilitation program on postural control and lower extremity function in individuals with 415 chronic ankle instability. Journal of Orthopaedic \& Sports Physical Therapy 37(6): 303-311.

416 Hassanlouei H, Arendt-Nielsen L, Kersting UG, Falla D. 2012. Effect of exercise-induced 417 fatigue on postural control of the knee. Journal of Electromyography and Kinesiology 22(3): 418 342-347.

419 Herrington L, Hatcher J, Hatcher A, McNicholas M. 2009. A comparison of Star Excursion 420 Balance Test reach distances between ACL deficient patients and asymptomatic controls. The 421 Knee 16(2): 149-152.

422 Hertel J, Braham RA, Hale SA, Olmsted-Kramer LC. 2006. Simplifying the star excursion 423 balance test: analyses of subjects with and without chronic ankle instability. Journal of 424 Orthopaedic \& Sports Physical Therapy 36(3): 131-137.

425 Hertel J, Miller SJ, Denegar CR. 2000. Intratester and intertester reliability during the Star 426 Excursion Balance Tests. Journal of Sport Rehabilitation 9(2): 104-116. 
427 Hewett TE, Myer GD, Ford KR. 2001. Prevention of anterior cruciate ligament injuries. 428 Current women's health reports 1(3): 218-224.

429 Holm I, Fosdahl MA, Friis A, Risberg MA, Myklebust G, Steen H. 2004. Effect of 430 neuromuscular training on proprioception, balance, muscle strength, and lower limb function in 431 female team handball players. Clinical Journal of Sport Medicine 14(2): 88-94.

432 Hubbard TJ, Kramer LC, Denegar CR, Hertel J. 2007. Correlations among multiple 433 measures of functional and mechanical instability in subjects with chronic ankle instability. 434 Journal of athletic training 42(3): 361.

435 Hutchinson MR, Laprade RF, Burnett QM, Moss R, Terpstra J. 1995. Injury surveillance at 436 the USTA Boys' Tennis Championships: a 6-yr study. Medicine and science in sports and 437 exercise 27(6): 826-831.

438 Ireland A, Maden-Wilkinson T, McPhee J, Cooke K, Narici M, Degens H, Rittweger J. 439 2013. Upper limb musclebone asymmetries and bone adaptation in elite youth tennis players 440 Medicine \& Science in Sports \& Exercise 45(9): 1749-1758.

441 Isles RC, Choy NL, Steer M, Nitz JC. 2004. Normal values of balance tests in women aged 442 20-80. Journal of the American Geriatrics Society 52(8): 1367-1372.

443 Khin-Myo-Hla, Ishii T, Sakane M, Hayashi K. 1999. Effect of anesthesia of the sinus tarsi on 444 peroneal reaction time in patients with functional instability of the ankle. Foot \& ankle 445 international 20(9): 554-559.

446 Kibler WB, Safran M. 2005. Tennis injuries. In Epidemiology of pediatric sports injuries. 447 Karger Publishers (Vol. 48, pp. 120-137).

448 Kinzey SJ, Armstrong CW. 1998. The reliability of the star-excursion test in assessing dynamic 449 balance. Journal of Orthopaedic \& Sports Physical Therapy 27(5): 356-360.

450 Knapik JJ, Cosiolima LM, Reynolds KL, Shumway RS. 2015. Efficacy of functional 451 movement screening for predicting injuries in coast guard cadets. Journal of Strength \& 452 Conditioning Research 29(5): 1157.

453 Kollock R, Hale D, Vogelpohl R, Kremer L, Cox C, Horner J, Allen M. 2018. The influence 454 of body armor on balance and movement quality. International Journal of Exercise Science 455 11(1): 648-656.

456 Kondrič M, Matković B, Furjan-Mandić G, Hadžić V, Dervišević E. 2011. Injuries in racket 457 sports among Slovenian players. Collegium antropologicum 35(2): 413-417.

458 Lam WK, Fan JX, Zheng Y, Lee WCC. 2018. Joint and plantar loading in table tennis topspin 459 forehand with different footwork. European journal of sport science 1-9.

460 Malliou VJ, Malliou P, Gioftsidou A, Pafis G, Katsikas C, Beneka A, ... Godolias G. 2008. 461 Balance exercise program before or after a tennis training session?. Journal of Back and 462 Musculoskeletal Rehabilitation 21(2): 87-90.

463 Miura K, Ishibashi Y, Tsuda E, Okamura Y, Otsuka H, Toh S. 2004. The effect of local and 464 general fatigue on knee proprioception. Arthroscopy: The Journal of Arthroscopic \& Related 465 Surgery 20(4): 414-418.

466 Munro AG, Herrington LC. 2010. Between-session reliability of the star excursion balance test. 467 Physical Therapy in Sport 11(4): 128-132. 
468 Murphy DF, Connolly DAJ, Beynnon BD. 2003. Risk factors for lower extremity injury: a

469

470

471

472

473

474

475

476

477

478

479

480

481

482

483

484

485

486

487

488

489

490

491

492

493

494

495

496

497

498

499

500

501

502

503

504

505

506

507

review of the literature. British journal of sports medicine 37(1): 13-29.

Noble BJ, Robertson RJ. 1996. Perceived exertion. Human Kinetics Publishers.

Noble BJ, Borg GA, Jacobs IRA, Ceci R, Kaiser P. 1983. A category-ratio perceived exertion scale: relationship to blood and muscle lactates and heart rate. Medicine and science in sports and exercise 15(6): 523-528.

Olmsted LC, Carcia CR, Hertel J, Shultz SJ. 2002. Efficacy of the star excursion balance tests in detecting reach deficits in subjects with chronic ankle instability. Journal of athletic training 37(4): 501.

Paterno MV, Myer GD, Ford KR, Hewett TE. 2004. Neuromuscular training improves singlelimb stability in young female athletes. Journal of Orthopaedic \& Sports Physical Therapy 34(6): 305-316.

Pau M, Ibba G, Attene G. 2014. Fatigue-induced balance impairment in young soccer players. Journal of athletic training 49(4): 454-461.

Plisky PJ, Rauh MJ, Kaminski TW, Underwood FB. 2006. Star Excursion Balance Test as a predictor of lower extremity injury in high school basketball players. Journal of Orthopaedic \& Sports Physical Therapy 36(12): 911-919.

Qian J, Zhang Y, Baker JS, Gu Y. 2016. Effects of performance level on lower limb kinematics during table tennis forehand loop. Acta of bioengineering and biomechanics 18(3): 149.

Rasool J, George K. 2007. The impact of single-leg dynamic balance training on dynamic stability. Physical therapy in sport 8(4): 177-184.

Ribeiro F, Santos F, Goncalves P, Oliveira J. 2008. Effects of volleyball match-induced fatigue on knee joint position sense. European Journal of Sport Science 8(6): 397-402.

Robinson R, Gribble P. 2008. Kinematic predictors of performance on the Star Excursion Balance Test. Journal of Sport Rehabilitation 17(4): 347-357.

Rozzi S, Yuktanandana P, Pincivero D, Lephart SM. 2000. Role of fatigue on proprioception and neuromuscular control. Proprioception and neuromuscular control in joint stability. Champaign, IL: Human Kinetics 2000: 375-383.

Sanchis-Moysi J, Dorado C, Olmedillas H, Serrano-Sanchez JA, Calbet JA. 2010. Bone and lean mass inter-arm asymmetries in young male tennis players depend on training frequency. European Journal of Applied Physiology 110 (1): 83-90.

Voight ML, Hardin JA, Blackburn TA, Tippett S, Canner GC. 1996. The effects of muscle fatigue on and the relationship of arm dominance to shoulder proprioception. Journal of Orthopaedic \& Sports Physical Therapy 23(6): 348-352.

Weaver NL, Mueller FO, Kalsbeek WD, Bowling JM. 1999. The North Carolina high school athletic injury study: design and methodology. Medicine and science in sports and exercise 31(1): 176-182.

Winter DA, Patla AE, Frank JS. 1990. Assessment of balance control in humans. Medical progress through technology 16(1-2): 31-51. 
508 Yaggie J, Armstrong WJ. 2004. Effects of lower extremity fatigue on indices of balance. 509 Journal of Sport Rehabilitation 13(4): 312-322.

510 Ye J, Sun D, Fekete G. 2018. Ba Duan Jin Preliminary Analysis of the Second Type of Plantar 511 Pressure. Physical Activity and Health, 2(1), 1-7.

512 Yu C, Shao S, Baker JS, Gu Y. 2018. Comparing the biomechanical characteristics between 513 squat and standing serves in female table tennis athletes. PeerJ 6: e4760.

514 Zakas A. 2006. Bilateral isokinetic peak torque of quadriceps and hamstring muscles in 515 professional soccer players with dominance on one or both two sides. Journal of Sports Medicine 516 and Physical Fitness 46(1): 28.

517 Zhang Z. 2017. Biomechanical analysis and model development applied to table tennis forehand 518 strokes. Doctoral dissertation, Loughborough University. 
Figure 1

Participants undertook the test procedures consisting of warm-up (W), rest (R), different training phases (Phasel, Phasell, Phaselll) and test (T), and end of test (E).

\section{Exercise Intensity}

Very, Very Light

Very Light

Light

Moderate

Hard

Very Hard

Maximal

\section{RPE}

$$
\begin{gathered}
6 \text { - } 8 \\
9 \text { - } 10 \\
11 \text { - } 12
\end{gathered}
$$

$$
13-14
$$

15 - 16

17 - 18

19 - 20 
Figure 2

The RPE scale for the study

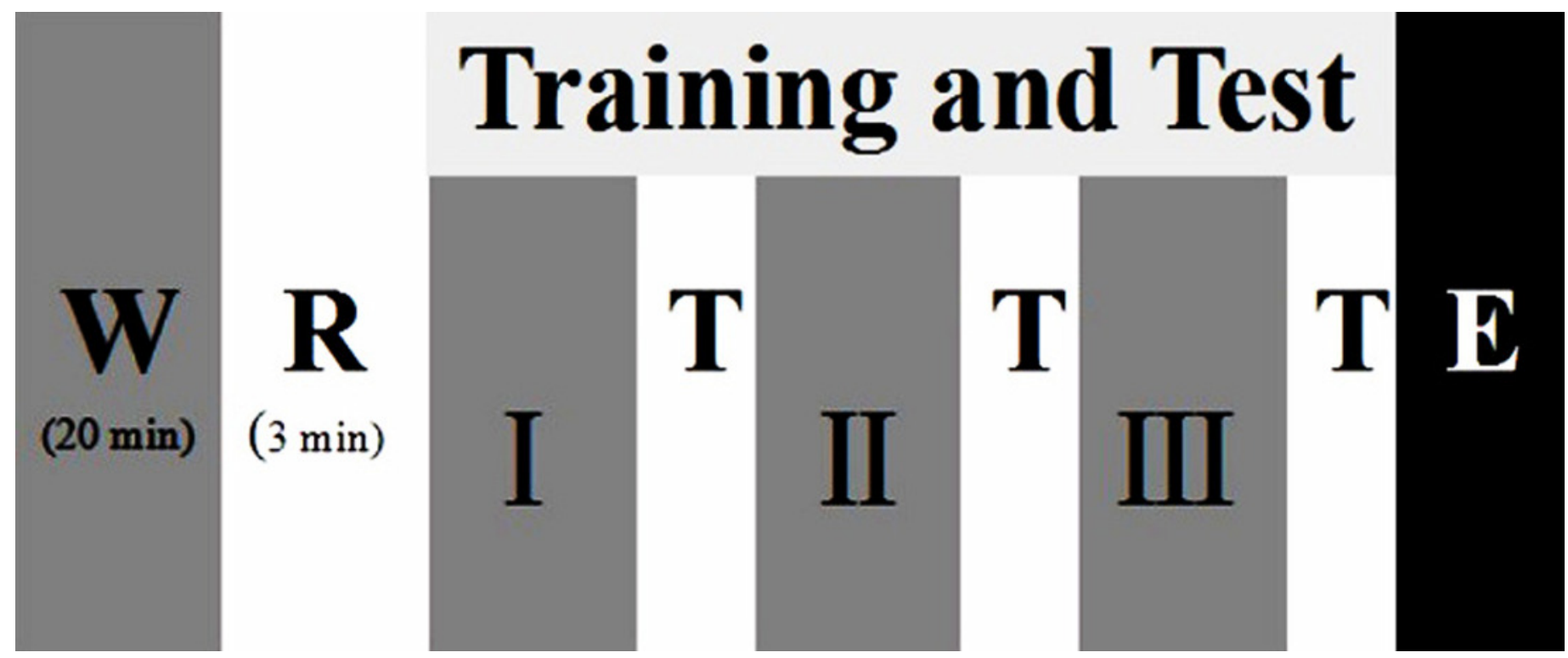


Figure 3

Reaching directions on the SEBT. Note: (A) and (B) show the performance for left- and right-leg stances, respectively.
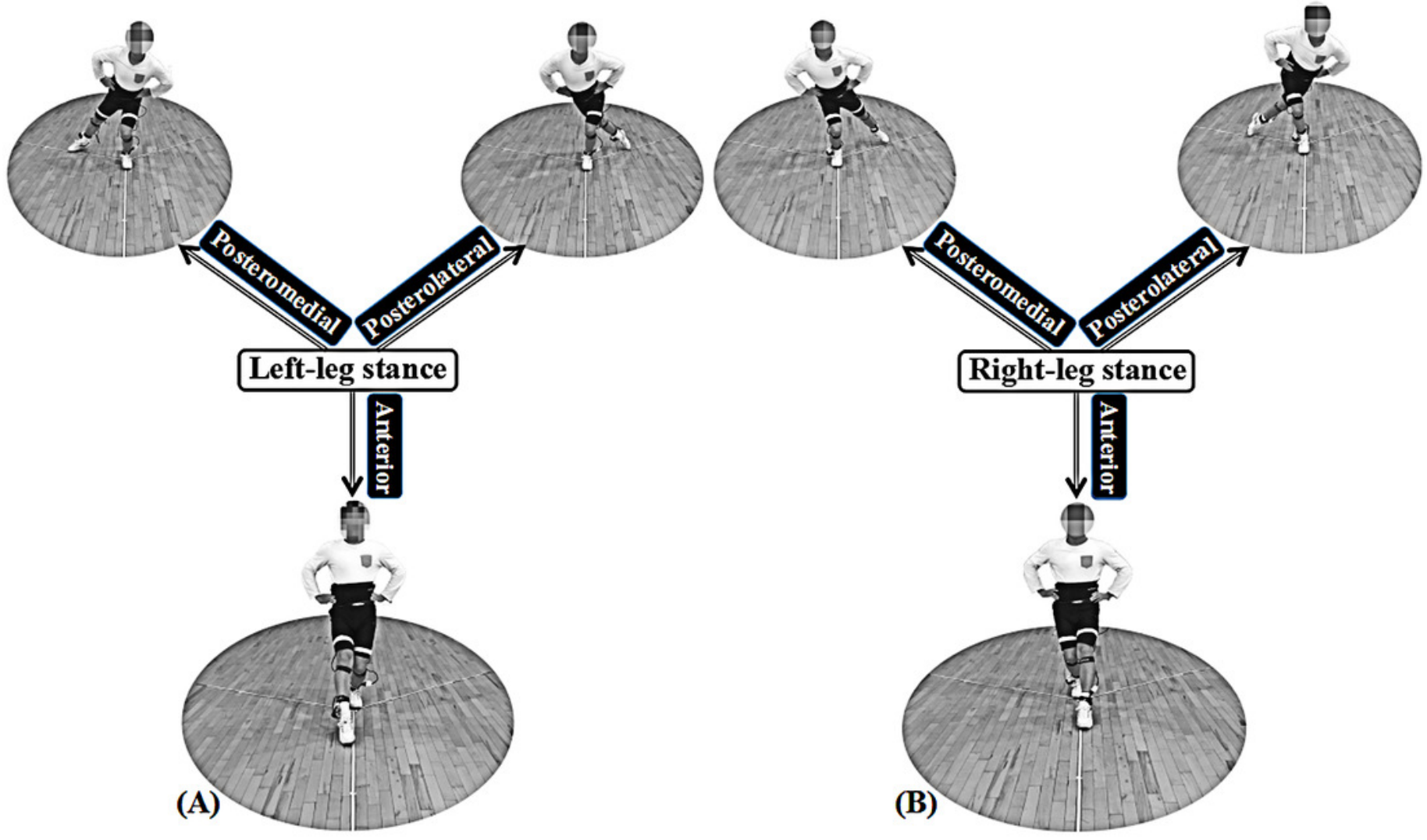
Figure 4

Trend of RPE for the male (A) and female (B) during the multi-ball training in each phase.

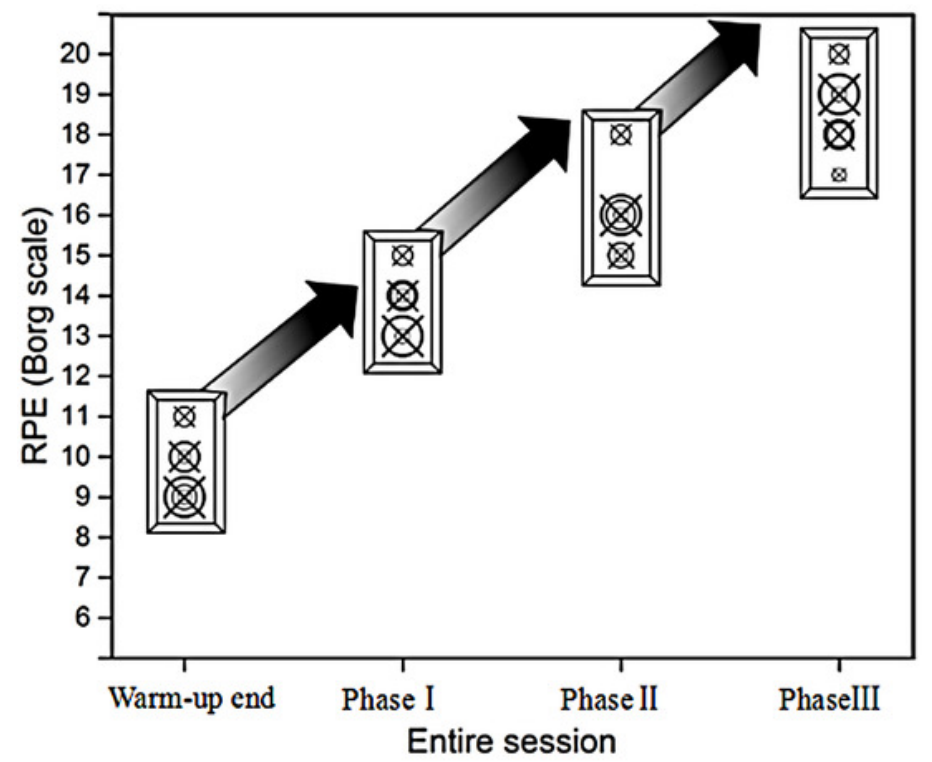

(A)

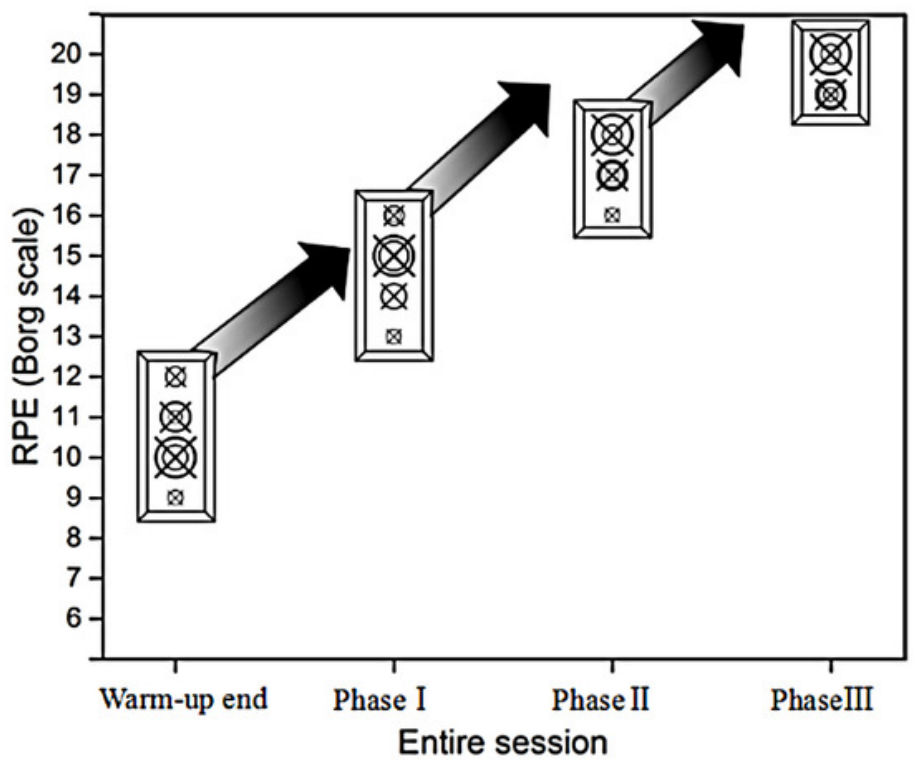

(B) 
Figure 5

The comparison of entire trend in the male (A) and female (B).

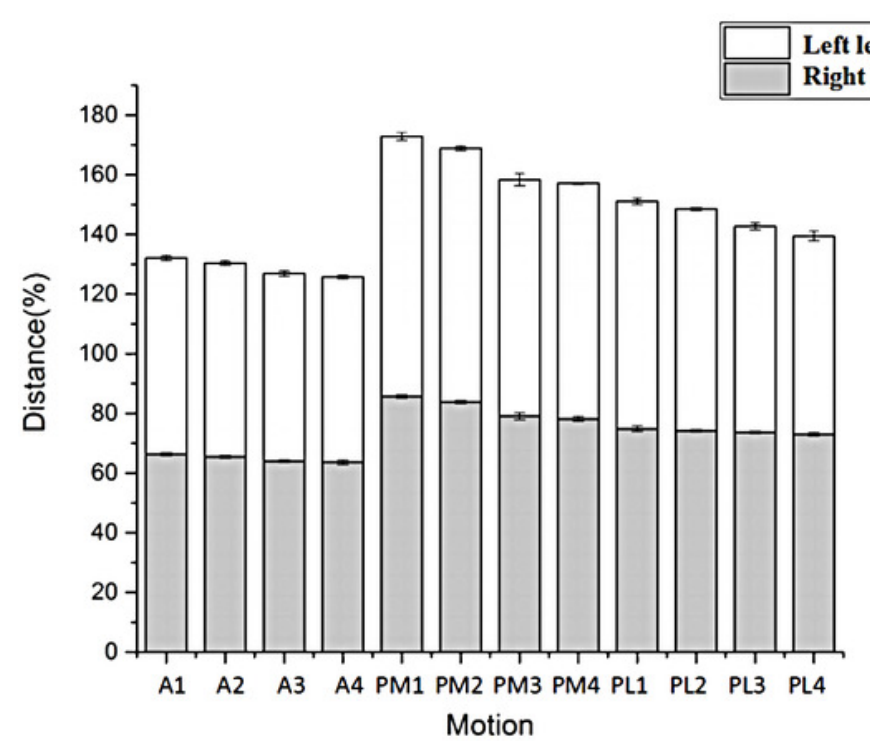

(A)

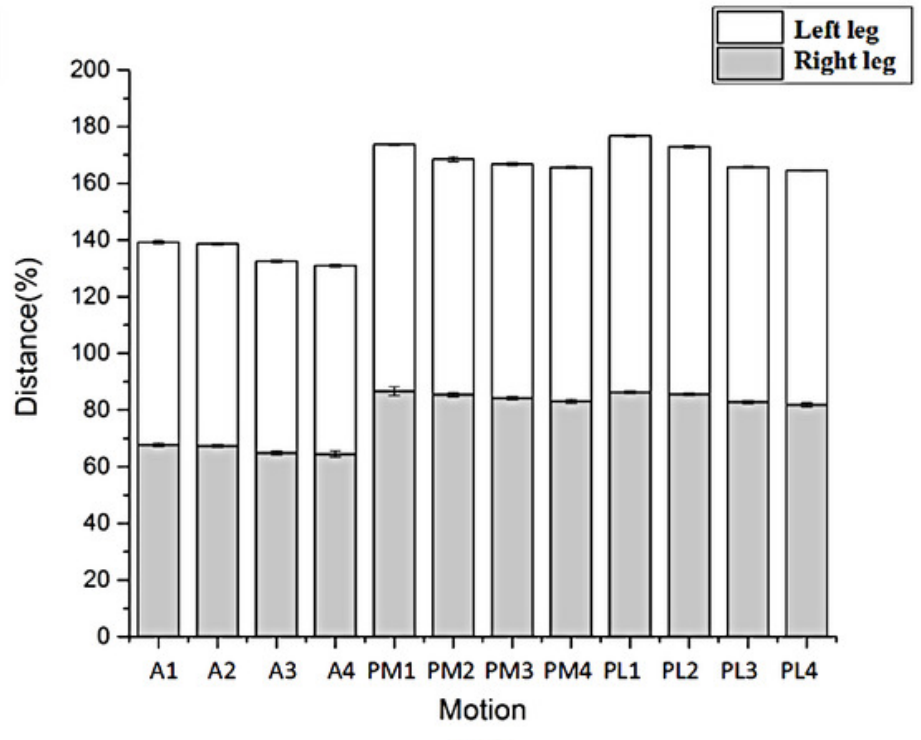

(B) 
Figure 6

The length of COP motion in each phase . Note: " " means significant difference began to show in COP length-X or $-Y$ compared with warm-up end.
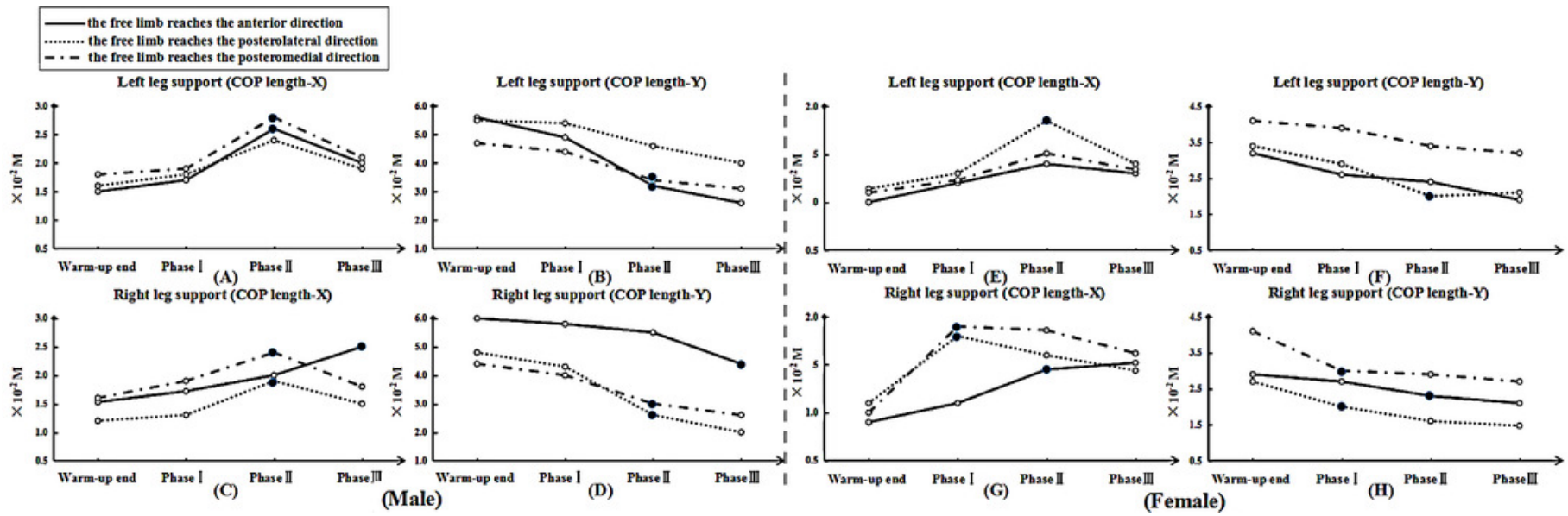


\section{Table $\mathbf{1}$ (on next page)}

Mean \pm standard deviations (mean \pm SD), standard error of measurement (SEM), 95\% confidence intervals $(\mathrm{Cl})$, effect sizes (ES) for the RPE values at warm-up end, phase I, phase II and phase III. 
1 Table 1: Mean \pm standard deviations (mean \pm SD), standard error of measurement (SEM), 95\% 2 confidence intervals (CI), effect sizes (ES) for the RPE values at warm-up end, phase I, phase II and 3 phase III.

\begin{tabular}{clcccc}
\hline Phase & Gender & Mean \pm SD & SEM & CI & ES \\
\hline \multirow{2}{*}{ Warm-up end } & Male & $9.67 \pm 0.82$ & 0.33 & $(8.81,10.52)$ & -- \\
& Female & $10.50 \pm 1.05$ & 0.43 & $(9.40,11.60)$ & -- \\
\multirow{2}{*}{ Phase I } & Male & $13.83 \pm 0.75$ & 0.31 & $(13.04,14.62)$ & $\S 0.93$ \\
& Female & $14.83 \pm 1.17$ & 0.48 & $(13.61,16.06)$ & $\S 0.89$ \\
& Male & $16.00 \pm 1.10$ & 0.45 & $(14.85,17.15)$ & $\S 0.95$ \\
Phase II & & & & & $\S 0.75$ \\
& Female & $17.17 \pm 0.75$ & 0.31 & $(16.38,17.96)$ & $\S 0.96$ \\
& & & & & $\S 0.77$ \\
& & & & & $¥ 0.97$ \\
& Male & $18.50 \pm 1.05$ & 0.43 & $(17.40,19.60)$ & $¥ 0.93$ \\
& & & & & $※ 0.75$ \\
& & & & & $\S 0.98$ \\
& Female & $19.33 \pm 0.52$ & 0.21 & $(18.79,19.88)$ & $¥ 0.92$ \\
& & & & & $※ 0.86$ \\
\hline
\end{tabular}

\section{Note:}

$5 \S$ shows a comparison with warm-up end $(p \leq 0.05)$.

$6 ¥$ shows a comparison with phase I $(p \leq 0.05)$.

7 ※ shows a comparison with phase $\mathbb{I}(p \leq 0.05)$.

8

9

10 
Table 2 (on next page)

Distance on the SEBT for each direction (mean \pm SD). 
1

2

3

4

5

8

8

Table 2: Distance on the SEBT for each direction (mean \pm SD).

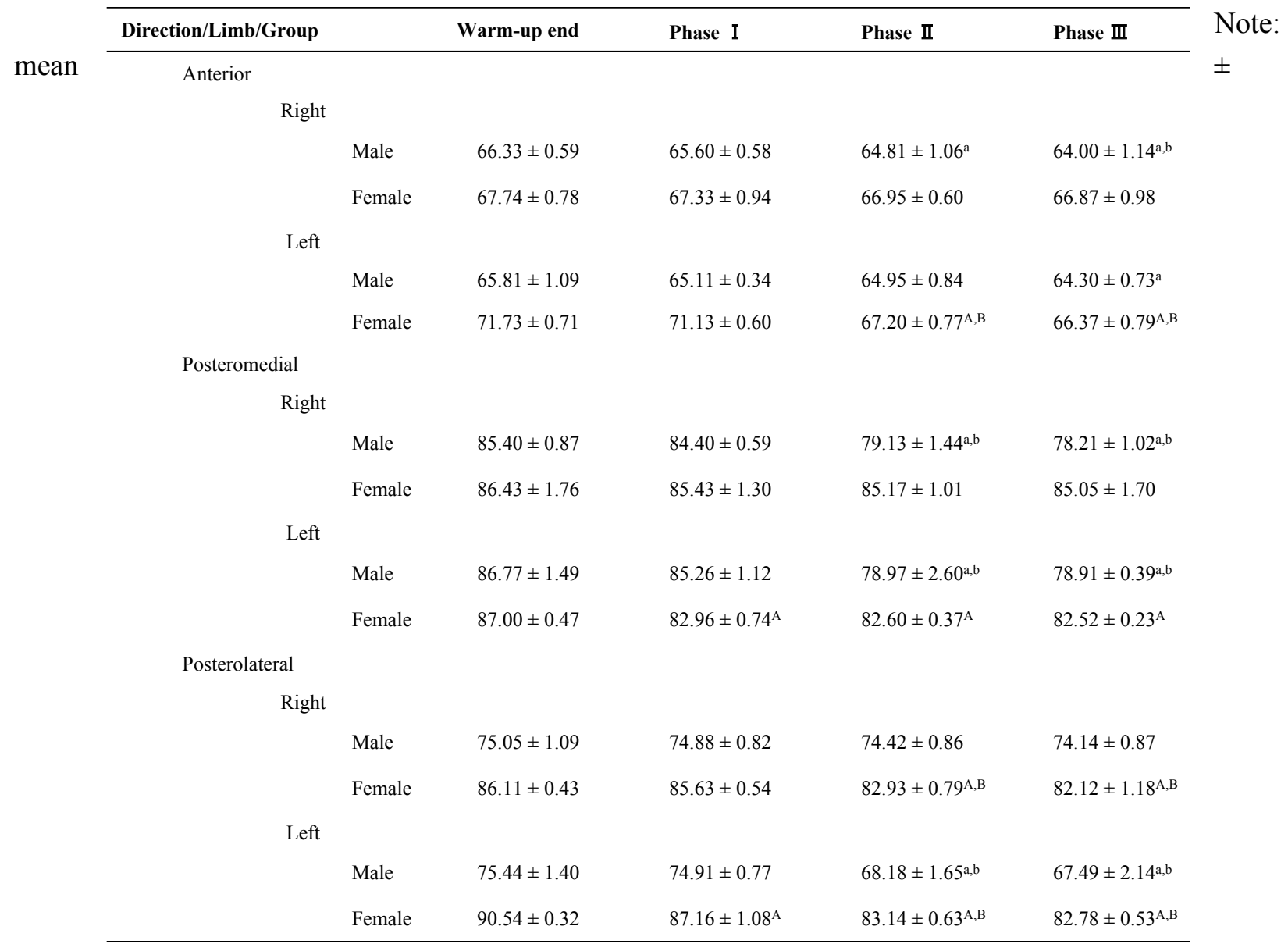

10 standard deviations are normalised reach distance (reach distance/leg length $\times 100$ ).

$11{ }^{\mathrm{a}}$ and ${ }^{\mathrm{A}}$ significant difference from warm-up end in male and female $(p \leq 0.05)$, respectively.

${ }^{\mathrm{b}}$ and ${ }^{\mathrm{B}}$ significant difference from phase $\mathrm{I}$ in male and female $(p \leq 0.05)$, respectively.

13

${ }^{\mathrm{c}}$ and ${ }^{\mathrm{C}}$ significant difference from phase $\mathrm{I}$ in male and female $(p \leq 0.05)$, respectively. 


\section{Table 3(on next page)}

Standard error of measurement (SEM), 95\% confidence intervals (CI), effect sizes (ES) for trials of males in each direction. 
3 Table 3: Standard error of measurement (SEM), 95\% confidence intervals (CI), effect sizes (ES) for 4 trials of males in each direction.

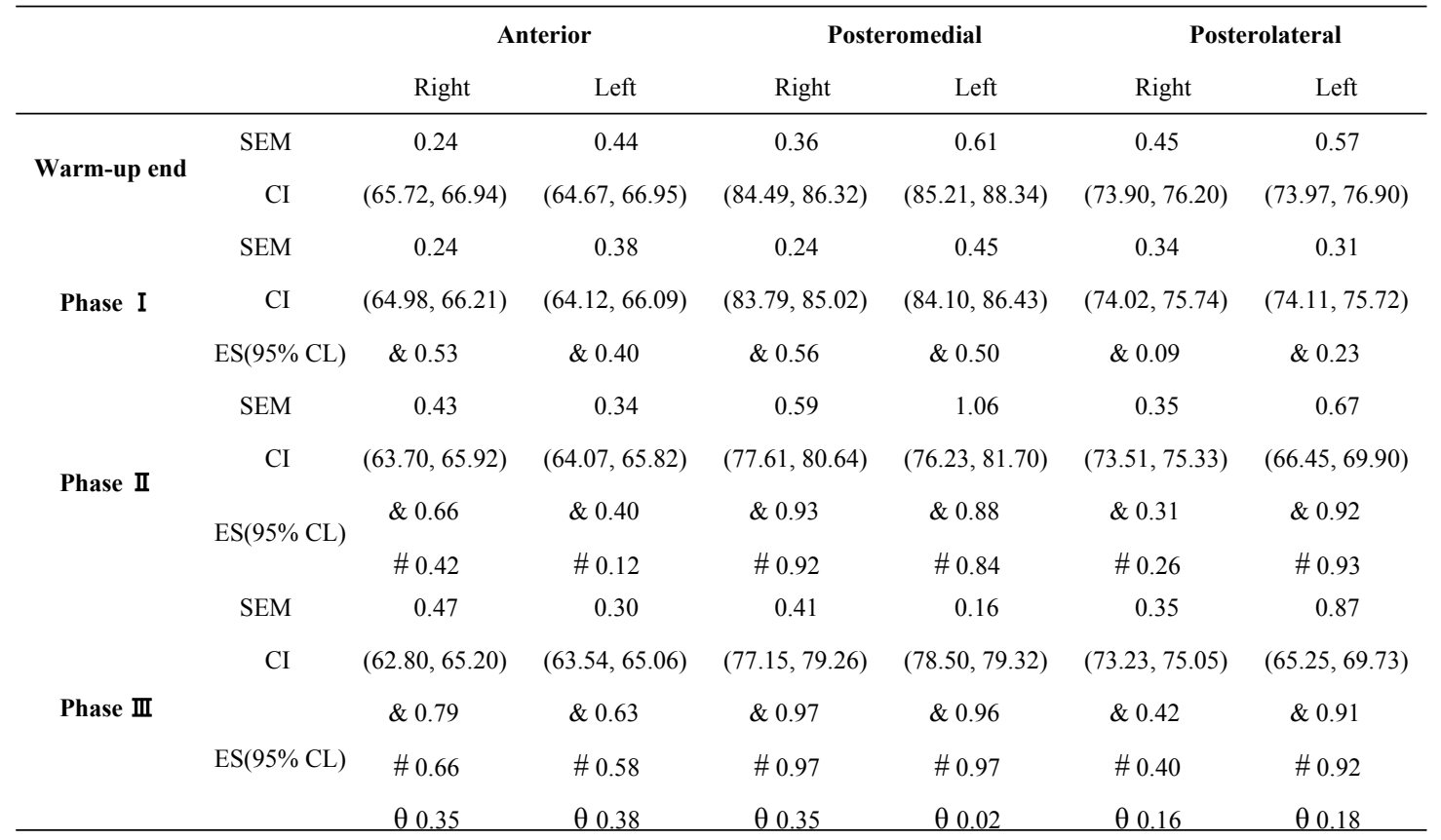

5 Note:

6 all values are normalised reach distance (reach distance/leg length $\times 100$ ).

$7 \quad \&$ shows a comparison with warm-up end $(p \leq 0.05)$.

8 \# shows a comparison with phase I $(p \leq 0.05)$.

$9 \theta$ shows a comparison with phase $\mathbb{I}(p \leq 0.05)$.

10

11

12 


\section{Table 4 (on next page)}

Standard error of measurement (SEM), 95\% confidence intervals (CI), effect sizes (ES) for trials of females in each direction. 
2 Table 4: Standard error of measurement (SEM), 95\% confidence intervals (CI), effect sizes (ES) for

3 trials of females in each direction.

\begin{tabular}{cccccccc}
\hline & & \multicolumn{2}{c}{ Anterior } & \multicolumn{2}{c}{ Posteromedial } & \multicolumn{2}{c}{ Posterolateral } \\
& & Right & Left & Right & Left & Right & Left \\
\hline \multirow{2}{*}{ Warm-up end } & SEM & 0.32 & 0.29 & 0.72 & 0.20 & 0.18 & 0.13 \\
& CI & $(66.92,68.57)$ & $(70.99,72.47)$ & $(84.58,88.28)$ & $(86.51,87.50)$ & $(85.66,86.56)$ & $(90.20,90.88)$ \\
Phase I & SEM & 0.38 & 0.24 & 0.53 & 0.30 & 0.22 & 0.44 \\
& CI & $(66.34,68.31)$ & $(70.51,71.75)$ & $(84.07,86.79)$ & $(82.18,83.73)$ & $(85.07,86.19)$ & $(86.03,88.30)$ \\
& ES(95\% CL) & $\& 0.23$ & $\& 0.42$ & $\& 0.31$ & $\& 0.96$ & $\& 0.44$ & $\& 0.90$ \\
Phase II & SEM & 0.24 & 0.31 & 0.41 & 0.15 & 0.32 & 0.26 \\
& CI & $(66.32,67.57)$ & $(66.40,68.01)$ & $(84.12,86.22)$ & $(82.21,82.99)$ & $(82.10,83.76)$ & $(82.47,83.80)$ \\
& ES(95\% CL) & $\& 0.49$ & $\& 0.95$ & $\& 0.40$ & $\& 0.98$ & $\& 0.93$ & $\& 0.99$ \\
& SEM & $\# 0.23$ & $\# 0.94$ & $\# 0.11$ & $\# 0.29$ & $\# 0.89$ & $\# 0.92$ \\
Phase III & 0.40 & 0.32 & 0.70 & 0.95 & 0.48 & 0.22 \\
& CI & $(65.84,67.89)$ & $(65.54,67.20)$ & $(83.26,86.84)$ & $(82.28,82.76)$ & $(80.88,83.35)$ & $(82.22,83.33)$ \\
& ES(95\% CL) & $\# 0.23$ & $\# 0.96$ & $\# 0.12$ & $\# 0.37$ & $\# 0.89$ & $\# 0.93$
\end{tabular}

\section{Note:}

5 all values are normalised reach distance (reach distance/leg length $\times 100$ ).

$6 \&$ shows a comparison with warm-up end $(p \leq 0.05)$.

7 \# shows a comparison with phase I $(p \leq 0.05)$.

$8 \boldsymbol{\theta}$ shows a comparison with phase II $(p \leq 0.05)$.

9 